\title{
Study on Efficacy and Safety of Shi's Manual Therapy Treatment for Lumbar Disc Herniation with Radiculopathy: Study Protocol for A Multicenter Randomized Controlled Trial
}

Huihao Wang

Shuguang Hospital https://orcid.org/0000-0001-7621-3916

Yuan Weian

Shuguang Hospital

Yu Zhongxiang

Shuguang Hospital

Wang Xiang

Shuguang Hospital

Zhao Xinxin

Tongji University

Deng Zhen

Shuguang Hospital

Shen Zhibi

Shuguang Hospital

Zhan Hongsheng ( 13918449223@139.com)

Shuguang Hospital

Study protocol

Keywords: lumbar dis version c herniation with radiculopathy, Shi' manual therapy, percutaneous endoscopic lumbar discectomy, protocol, multicenter randomized controlled trial.

Posted Date: June 28th, 2021

DOI: https://doi.org/10.21203/rs.3.rs-197349/v1

License: (1) This work is licensed under a Creative Commons Attribution 4.0 International License. Read Full License 


\section{Abstract \\ Background}

Lumbar disc herniation (LDH) is a common chronic musculoskeletal disorder that seriously affects quality of life. Percutaneous endoscopic lumbar diskectomy (PELD) technique was developed to address spine nerve root compression by direct visualization of pathological findings while minimizing tissue destruction upon exposure. It is an effective and safe treatment for LDH. However, recurrent LDH is a major concern after lumbar discectomy for primary LDH. A considerable number of clinical studies reported that patients will benefit from manual therapy practice for LDH with radiculopathy patients. Shi's manual therapy (SMT) is established based on the Traditional Chinese medicine (TCM) theory, which has been shown to have a superior effect in alleviating muscle tension and loosen joints to improve lumbar and leg pain, radiculopathy, stiffness, activity discomfort, and related disorders. But there is a lack of high-quality clinical evidences to support the conclusion. The purpose of this study will be to evaluate the efficacy and safety of the combination of Shi' manual therapy (SMT) for reducing the recurrent rate of LDH with radiculopathy.

\section{Methods/design:}

A multicenter randomized controlled trial (RCT) with a 1years' follow-up period will be performed. A total of 510 participants with LDH with radiculopathy will be recruited in four clinical centers. Sample size estimation was calculated and statistical analysis will be performed calculated and supervised by biostatisticians from the independent third-party research institution. Two hundred and fifty-five subjects will be randomly allocated to each group. The subject in the control group will undergo the PELD. Participants in the intervention group will be treated with SMT, and the straight leg raising angle will be evaluated. If the patients' straight leg raising the angle of the affected side is close to the healthy side, and the treatment is finished, if not, the PELD procedure will proceed. The primary outcome will be the recurrence rate of LDH with radiculopathy and the ODI score. The secondary outcome measures will be the VAS score for the pain of lumbar and lower limbs, the straight leg raising angle, the stability of the operated lumbar segment, and SF-36 scores. Assessments will occur at baseline, post-operation, and at 1 week, 4 weeks, 13 weeks, 26 weeks and 1year post-operation. In addition, adverse events related to clinical symptoms and signs and results of laboratory tests will be documented during the clinical trials.

\section{Discussion}

This study will provide reliable evidence of the effectiveness and safety of the combination of SMT and PELD for LDH with radiculopathy. If the results are favorable, it is expected that the patients with LDH with radiculopathy will benefit from this study, many patients may have a good alternative treatment for LDH with radiculopathy. 


\section{Trial registration:}

ChiCTR, ChiCTR2000036515. Registered on 13 November 2020.

\section{Background}

Herniated lumbar disc is a displacement of disc material (nucleus pulposus or annulus fibrosis) beyond the intervertebral disc space[1], with a $70 \%$ overall prevalence rate

in developed countries[2]. The diagnosis can be confirmed by radiological examination. However, MRI findings of herniated disc are not always accompanied by clinical symptoms[3]. Conservative treatments are first-line treatments for lumbar disc herniation (LDH) include medication, bed rest, physical therapy, massage, kinesitherapy, manual therapy, and traction therapy. Manual therapy related to clinical massage, spinal manipulation (SM), or mobilization, which is applied to release the joint fixation of the involved segment and to restore the flexibility of the lumbar spine to improve the biomechanical environment of the affected nerve roots on LDH patients. A review reported that SM is otherwise an alternative or complementary medical method, though with limited curative effect, and only better than placebo if there is no severe adverse effect in its performance[4]. In the North American Spine Society's (NASS) Evidence-Based Clinical Guideline for the Diagnosis and Treatment of LDH with radiculopathy, spinal manipulation is the grade of recommendation: $\mathrm{C}$. There is an insufficient evidence to make a recommendation for or against the use of spinal manipulation compared with chemonucleolysis in patients with lumbar disc herniation with radiculopathy and the grade of recommendation: I (insufficient evidence)[5].

Despite a lack of clear evidence-based medicine of its safety and efficacy, spinal manipulation is still popular in clinical treatment. A considerable number of clinical studies reported that patients will benefit from SM practice for LDH with radiculopathy patients. The former reports proved that an effective SM will load appropriate stress on the involved joints and produce non-noxious stimuli[6, 7]. The benefits are accompanied by potential, yet rare risks in terms of serious adverse events. Therefore, it is reasonable to use an 'appropriate mechanical intervention' to achieve a balance between releasing the tension of the joint and reducing the risk of injury during the SM performance.

Surgical intervention may become necessary if conservative therapeutic measures fail or paralysis is present. minimally invasive spine surgery has become increasingly used. Percutaneous endoscopic lumbar diskectomy (PELD) technique was developed to address spine nerve root compression by direct visualization of pathological findings while minimizing tissue destruction upon exposure. It is an effective and safe treatment for LDH, lumbar spinal stenosis, recurrent LDH, and other lumbar diseases. Operative times, blood loss, lengths of hospitalization, and need for postoperative pain medications have all generally been reduced with microendoscopic discectomy[8]. However, complications related to PELD include dural tear $(1.1 \%)$, intervertebral infection $(0.47 \%)$, incomplete removal of the disc $(2.77 \%)$, nerve root injury, recurrence, and so on[9]. Recurrent LDH is a major concern after lumbar discectomy for 
primary LDH, occurs in approximately $10.3 \%-17.6 \%$ of patients $[10,11]$. The clinical characteristics and risk factors of recurrent LDH, such as sex, obesity (body mass index $\geq 25$ ), older age ( $\geq 50$ years old), smoking history, etc., remain controversial[ $[12,13]$.

Shi's manual therapy (SMT) is established based on the TCM theory, which believed that the lumbar and leg complaints were induced by tendon off-position and joint subluxation (International Classification of Diseases 11th Revision, ICD-11). It is one of the characteristic techniques of Shi's traumatology TCM academic school with years' clinical experience summary and accumulation, and the details will be described below. The techniques with the stress that is rapidly loaded are also called 'manipulation' or 'thrusting adjustment' with a typical feature of high- velocity and low- amplitude (HVLA). According to the advanced research progress and our clinical observation, there is no obvious discomfort effect in the short term and has been no report of a severe adverse event in the short and middle term for SMT[14]. The main purpose of it is to alleviate muscle tension and loosen joints to improve uncomfortable symptoms, such as lumbar and leg pain, radiculopathy, stiffness, activity discomfort, and related disorders.

In our experience, it is more easily acceptable for patients to SMT than PELD in China. Therefore, the authors design an integrated cooperative treatment protocol. If our hypothesis is proven, it will provide a choice for patients with LDH with radiculopathy who prefer no surgery or an alternative and complementary option before receiving PELD. The participants will fully understand the benefits and risks before being recruited and signing the informed consent.

To the authors' knowledge, a detailed description of a multicenter RCT of integrated cooperative management with SMT and PELD for LDH with radiculopathy is not available in the related literature.

\section{Study aims}

This study aims to report a multicenter RCT study protocol to investigate the safety of the combination of SMT and PELD with PELD only for LDH with radiculopathy, and the superiority of reducing the recurrence rate.

The specific aim includes 1 ) to assess the effects of SMT for LDH with radiculopathy; 2 ) to report the superiority of reducing the recurrence rate of the combination of SMT and PELD for LDH with radiculopathy.

\section{Methods/design}

\section{Study setting}

Follow-up assessments with questionnaires will be conducted on day 1, at the recovery site and 1week, 4 weeks, 13 weeks, and 26weeks postoperatively; and will include the ODI, the VAS score for pain of lumbar and lower limbs, straight leg elevation angle of the affected side, and SF-36 health survey. Pre- and 
postoperative magnetic resonance imaging (MRI), computed tomography (CT), and X-ray films will be documented and obtained again at 4 weeks, 13 weeks, 26 weeks and 1year, respectively. The assessments will be performed by 2 physicians, who are not involved in the treatments.

A multicenter prospective RCT with unblinded treatment and blinded outcome assessment will be conducted in the in four clinical centers, Shanghai, China, including Shuguang hospital affiliated to Shanghai University of Traditional Chinese Medicine (TCM), Shanghai municipal TCM hospital affiliated to Shanghai University of TCM, Tenth people's hospital affiliated to Tongji University and Shanghai Baoshan Hospital of Integrated TCM. Sample size estimation was calculated and statistical analysis will be performed calculated and supervised by biostatisticians from the Clinical Epidemiology and Medical Statistics Center, Tongji University School of Medicine.

Two hundred and fifty-five participants of LDH with radiculopathy will be randomly allocated to each group. Participants in the control group will undergo the PELD in 0 3 days, and then receive oral NSAID (Diclofenac sodium sustained release tablets $75 \mathrm{mg} \mathrm{qd}$ ) as needed for $3 \sim 5$ days. Participants in the intervention group will be treated with SMT in 0 3 days, and the straight leg raising angle will be evaluated. If the patients' straight leg raising the angle of the affected side is close to the healthy side, and the treatment is finished, if not, the PELD procedure will proceed.

Follow-up evaluations were performed in the outpatient clinic. Treatment will cease if the participant perceives that the condition is adequately improved or if perioperative complications occur.

Physicians in charge will collect, assess, report, and manage solicited and spontaneously reported adverse events and other unintended effects of trial interventions or trial conduct to the supervising doctor.

The research team will make judgments and conduct medical treatment for free, which is inherent to this study. Important protocol modifications must be approved by all authors and the IRB of Shuguang Hospital Affiliated to Shanghai University of TCM must be informed to amend the ethics committee approval.

\section{Ethics approval}

The study design and procedures were approved by the IRB of Shuguang Hospital Affiliated to Shanghai University of TCM (Approval Number: 2020-850-57-01), and the procedures followed were following the Declaration of Helsinki from 1975, as revised in 2000, as revised in 2000.

Written informed consent will be obtained from all participants before enrollment in the study.

\section{Patient and Public Involvement}

Five hundred and ten participants of LDH with radiculopathy will be recruited in the inpatient department of the Shuguang Hospital from October 2020 to September 2022 and by referral from an orthopedic 
surgeon and a physiotherapist. A flow chart of trial participation is provided in Fig. 1. The flowchart for participant identification, inclusion, study design, interventions, assessments, and follow-up was shown in Table 1. The inclusion and exclusion criteria are summarized in Table 2.

The personal information about potential and enrolled participants will be collected by outpatient doctors. The outcome data will be retained in the custody of independent statistician custody, and all data will be aggregated and maintained by the supervising doctor to protect confidentiality after the trial.

The supervising doctor will have access to the final trial dataset and disclosure of contractual agreements that limit such access for investigators.

\section{Percutaneous Endoscopic Lumbar Diskectomy (PELD)}

A senior physiotherapist with medical doctorate qualifications and a minimum of 10 years of experience will operate. PELD procedures will be performed as followed[15]:

1. Anesthesia and positioning: PELD by the transforaminal approach is performed under local anesthesia ( $20 \mathrm{ml} 1 \%$ lidocaine and $20 \mathrm{ml}$ normal saline) with the patient in the lateral position or prone position on a radiolucent operating table;

2. Surgical technique: Axial MRI or CT is used to get an approximate idea of the distance of the skin entry point from the midline. The needle trajectory is planned to target the ruptured fragment while avoiding the contents of the peritoneal sac. After satisfactory anesthesia with the patient, the surgeon routinely performs routine disc puncture and staining and then expands the working channel step by step according to Young's technique (YESS) and Maxmore technique. C-arm X-ray machine fluoroscopy confirms that the pipeline is placed in a good position. Then, the pipeline is connected to the irrigation device and the light source, and the surgeon sequentially performs ventral, dorsal, cranial, and caudal bony and soft decompression with laser and forceps on the patient's target intervertebral disc segment, and the contralateral and lateral recesses are gradually decompressed. After that, the surgeon observes the pulsation state of the nerve root of the dural sac, cough test, and straight leg raising test to evaluate the degree of spinal canal decompression until satisfaction. Finally, the surgeon draws out the irrigation fluid in the patient's intervertebral disc space, injects steroid drugs ( $1 \mathrm{ml}$ of Depot), sutures the wound, and completes the operation.

3. Postoperative management of the patients will include『a) a simple adhesive bandage was on the stitch and the suture would removal after $10 \sim 14$ days; b) a lumbar brace was required to wear for six weeks, and avoided heavy physical work and sitting for long periods for three months; and c) oral Celebrex capsule qd would be received as needed for $0 \sim 3$ days.

\section{Shi's manual therapy (SMT)}

A senior physiotherapist with medical doctorate qualifications and a minimum of 25 years of experience in SMT will perform the interventions on the participants. Participants in the intervention group will be treated with SMT in 0 3 days, and the straight leg raising angle will be evaluated. If the patients' straight 
leg raising the angle of the affected side is close to the healthy side, and the treatment is finished, if not, the PELD procedure will proceed.

The key procedure of SMT includes the following steps mainly:

1. Anesthesia and positioning: SMT is performed under local anesthesia $(20 \mathrm{ml} 1 \%$ lidocaine and $20 \mathrm{ml}$ normal saline) with the patient at the target disc segment nerve root outlet in the supine position on a radiolucent operating table;

2. Bending hips and knees, pressing and stretching techniques: the patient lies in the supine position. The therapist presses both lower limbs with hips and knees, and then pulls and stretches the affected leg in various directions several times, and then rotates left and right to pull and stretch the affected leg several times;

3. Straight leg raises and compression techniques: the assistant presses the patient's normal hip joint, the therapist presses the knee with one hand and the ankle with the other, lifts the patient's lower limbs, gradually raising it to $80^{\circ} \sim 90^{\circ}$, repeating 10 15 times. And then, the therapist stands on the normal side of the patient and raises the patient's legs in a back-holding style. The therapist holds the patient's feet with his hands over his head and puts the therapist's back against the patient's knee joint to prevent flexion. After the therapist raises the patient's affected limb to the limit range, performs foot extension exercises passively 3 5 times;

4. Lumbar spine oblique pulling technique in lateral position: the patient lies on the affected side, and the therapist straightens the patient's lower limbs and flexes the hips and knees of the upper limbs. And then the therapist presses the patient's shoulder joint outward and pushes down, while the other hand fixes the sacroiliac joint and presses inwardly. It is advisable to hear a "click" sound from the waist. It is the same procedure that will be used on the other side. The treatment will be stopped if the patients feel uncomfortable or for any other reasons, but the recorded data will be analyzed statistically.

\section{Assessment and follow-up}

Participants will receive a serial preoperative radiographic evaluation, with routine anteroposterior (AP), lateral and dynamic radiographic views, MRI, and CT scan to determine the target level in conjunction with a thorough clinical history and physical examination. Then, diagnostic selective nerve root blocks or electromyography (EMG) and nerve conduction studies will be assessed for the individual's radiculopathy origins[16].

The first examination will be conducted before the operation for baseline assessment (visit 0). Then, assessments will be conducted on the second-day post-operation (visit 1), and at 1week (visit 2), 4 weeks (visit 3), 13 weeks(visit 4), 26 weeks (visit 5) and 1year (visit 6) post-operation. The primary and secondary outcomes and possible complications will be recorded. All participants will respond to the ODI questionnaire and pain scoring for the lumbar and lower limbs with the VAS evaluation. Besides, CT, MRI, and X-ray imaging, including AP, lateral, and dynamic (hyperextension and hyperflexion) radiographic 
views assessments will be conducted. The radiation exposure dose is safe for all participants which are assessed by two radiologists. Note that the Dynamic X-ray imaging will be carried in visit 0 , visit 4 , visit 5 and visit 6 , while MRI and CT scan will be carried in visit 0 , visit 1 , visit 5 , and visit 6 .

\section{Randomization}

An independent statistician will perform the subject enrollment and intervention assignment. The randomization will be carried out before the subjects assigning to groups in four independent clinical centers. After suitable participants are filtered by the acceptance criteria, their basic information will be transmitted to the statistician. He will produce a computer-generated randomization sequence that will be placed in sequentially numbered opaque sealed envelopes. The randomization sequence will contain equal numbers of participants in each group. Due to the nature of the interventions, the participants were not blinded to the treatment group.

\section{Outcome measures}

\section{Primary outcome}

1) Recurrence rate

The recurrence rate of LDH with radiculopathy at 1 year post-operation between two groups. Clinically speaking, herniation at the same level and same side would be more appropriate for recurrence. If the symptom returns after a pain-free period, it can be defined as recurrence[17].

2) Oswestry Disability Index (ODI)

And the Simplified Chinese-Mandarin Chinese version of the ODI will be applied for evaluating the degree of low back pain and disability[18].

\section{Secondary outcomes}

1) The severity of lumbar and lower limbs (VAS)

VAS has also been used in many studies to measure pain as the main complaint[19] and has shown high reliability and validity. The low back pain and lower limbs pain will be evaluated with a 10-point scale: 0 as painless and 10 as extremely painful. The patients will be asked to provide an average pain level over the previous 24 hours.

2) Straight leg raising angle of the affected side

A protractor will be used to measure the straight leg angle of the healthy side and the affected side to access the efficacy of the treatment, and to calculate the difference in both sides. The straight leg raise test also called the Lasegue test, is a fundamental neurological maneuver during the physical 
examination of the patient with lower back pain aimed to assess the sciatic compromise due to lumbosacral nerve root irritation[20].

3) Stability of the lumbar spine

Radiological parameters will be measured, including lumbar curvature (L1-S1, tangential method) in neutral position with Harrison posterior tangent method and the segmental Cobb angle of hyperextension and hyperflexion, on the X-ray films at the operative level (SA)[21]. The radiographic instability is defined as evidence of translational motion at one spinal motion segment more than $3 \mathrm{~mm}$ in the lumbar spine or $5 \mathrm{~mm}$ at L5-S1 or as angulation of one motion segment more than $10^{\circ}$ on lateral flexion-extension radiographs[22].

4) Short Form 36 version 2 (SF-36v2) Health Survey scores

We used the Singapore English and Chinese language version of the SF-36v2 questionnaire to document the health-related quality of life. The version includes 36 self-administered questions which examining 8 dimensions of the participant's general health, including physical functioning, physical roles, bodily pain, general health, vitality, social functioning, emotional roles, and mental health, with higher scores (range, 0100) reflecting better-perceived health[23].

\section{Quality assurance}

To ensure that interventions are of a high standard and delivered following the trial protocol, surgeons and physiotherapists responsible for performing the interventions of SMT and PELD will attend a two-day training workshop on the delivery of the intervention programs. They will also be provided with a written protocol and standardized recording documents. Besides, all interventions provided to patients will be carefully recorded.

\section{Data analysis}

\section{Sample size calculation}

The recurrence rate of LDH with radiculopathy at 1 year post-operation will be the primary outcome measure for sample size calculations, as reported by a previously published trial at $17.6 \%{ }^{11}$. Independent $t$-tests will be utilized to detect clinical differences between the means of continuous variables (primary outcomes), with $80 \%$ power and alpha set at 0.025 . Assuming that recurrence rate in the intervention group (effect size $=8.6$; standard deviation $=2.5$ ) will be reduced by $9 \%$ compared with the control group (effect size $=17.6$; standard deviation $=2.5$ ). Besides, a dropout rate of $16 \%$ will also be taken into account. Estimations indicated that 255 individuals per group are required. Hence, a minimum of 510 patients will be necessary for the sample size.

\section{Statistical methods}


Baseline characteristics will be summarized per group using the number of observations, mean, standard deviation, median, minimum, and maximum for continuous measures and using the number of observations and frequency for categorical measures. The primary and secondary outcome measures are either continuous or ordinal and will be analyzed using generalized linear mixed models. For instance, the outcome variables will be the ODI and the predictors will be time, treatment group, and an interaction term for the time by treatment group. The effects of these protocols will be tested through both intention-totreat and per-protocol analysis. For the intention-to-treat analysis, missing post-treatment or follow-up outcome data will be replicated from previous measures available (by assuming no change for noncompleters). For the per-protocol analysis, data from excluded subjects will be disregarded for analysis. The groups will be compared at baseline by the chi-square test for qualitative data and by the $t$-test for quantitative data. To analyze changes in outcomes at baseline, post-treatment, and follow-up between and within groups, analysis of variance with repeated measures will be applied. A normality test will be applied to the outcome measures; when data are not normally distributed, equivalent nonparametric tests will be used. The primary and secondary outcome measures will also be compared between treatment groups at each time point using independent t-tests. The results will be presented as percentages for categorical variables and as the means, medians, standard deviations, and $95 \%$ confidence intervals for continuous variables. Data analyses will be performed using SPSS 22.0 for Windows version 18.0 (SPSS Inc., Chicago, IL, USA) and the significance value for all tests will be set at $P<0.05$.

\section{Controlling bias}

It should be noted that the number of subjects assigned to Shuguang hospital affiliated to Shanghai University of TCM is double that of the other centers. Because the Shuguang hospital is divided into two independent branches, including the different doctors, inpatient department and operating room, which can be regarded as two sub-centers. Therefore, the number of subjects in all sub-centers is balanced.

To minimize bias randomization, concealed allocation, specific inclusion and exclusion criteria, blinded outcome assessment, blind data analysis, and intention to treat analysis have been used. It was not possible to blind the surgeons and therapists to perform the interventions.

The independent statistician will audit trial conduct every 12 weeks, and report directly to the superior doctor.

\section{Discussion}

This protocol outlines the rationale and design for a multicenter RCT to investigate the safety of the combination of SMT and PELD with PELD only for LDH with radiculopathy and the superiority of reducing the recurrence rate in 6 months.

The key factor affecting the quality of this trial is the quality control of the SMT and PELD given by the therapists and surgeons from 4 clinical centers. Even though the doctors may perform the same 
techniques of SMT and PELD, it is understandable that the operational details and personal habits might be different. It will be minimizing the possibility through the following aspects:

1. Before officially starts, every research participant in this project needs to obtain Good Clinical Practice (GCP) certificates authorized by China SFDA. It is to ensure that all aspects of research are standardized and to reduce bias during the implementation process.

2. To make the operation process as standardized and consistent as possible, the complete operation videos were recorded by a professional videographer for the SMT performed by a senior therapist and the PELD operated by an experienced surgeon in Shuguang Hospital. All SMT therapists and PELD surgeons in 4 clinical centers have to be trained and pass the assessment.

3. Only one senior SMT therapist and one PELD surgeon will be appointed to perform the interventions in one clinical center. All of them will not be allowed to perform the intervention until they have been trained and meet the requirements of this project.

It should be noted that Shuguang hospital affiliated to Shanghai University of TCM is divided into two independent branches, including the different doctors, inpatient departments, and operating rooms, which can be regarded as two sub-centers. Therefore, the number of subjects in all sub-centers is balanced.

Shi's orthopedics and traumatology academic school with manual therapy are well-known throughout China, with a history of 150 years, which is officially recognized by China's health care administration, which is significantly helpful in improving patient compliance in the study. However, the risk associated with spinal manipulation still needs to be noted. The subjects will be eligible for inclusion and exclusion criteria to minimize the occurrence of complications. Otherwise, the surgeon doctor will immediately perform PELD or routine open surgery, once the patient has adverse reactions after SMT such as nerve root damage or paralysis.

Statistical analysis is another challenging problem difficult to resolve. A multicenter prospective RCT will be conducted in four clinical centers and 255 participants will be randomly allocated to each group. Participants in the intervention group will be treated with SMT firstly, and the straight leg raising angle will be evaluated. However, we assume that if the patients' straight leg raising the angle of the affected side is close to the healthy side, and the treatment is finished, if not, the PELD procedure will proceed. The data will be calculated and analyzed through hierarchical statistics by an independent biostatistician.

This design of protocol has an advantage in the expertise of the physiotherapist. However, this may affect the generalizability of the conclusion. The results will contribute to evidence-based manual therapy leading to improved clinical decision making in this field of clinical practice.

The results of this trial will be published to the public.

\section{Abbreviations}

SMT 
Shi' manual therapy

PELD

percutaneous endoscopic lumbar discectomy

$\mathrm{LDH}$

lumbar disc herniation

RCT

a randomized controlled trial

TCM

traditional Chinese medicine

NSAID

the nonsteroidal anti-inflammatory drug

ODI

Oswestry Disability Index

SF-36v2

Short Form 36 version 2

VAS

the visual analog scale

MRI

magnetic resonance imaging

CT

computed tomography

EMG

electromyography

AP

anteroposterior radiographic views

\section{Declarations}

\section{Ethics approval and consent to participate}

The study design and procedures were approved by the Chinese Ethics Committee of Registering Clinical Trials (Protocol Number: ChiCTR2000036515), and the procedures followed were following the Helsinki Declaration of 1975 , as revised in 2000 . Written informed consent will be obtained from all participants before enrolment in the study.

\section{Consent for publication}

Consent for publication was obtained from the all authors.

\section{Availability of data and material}

Not applicable. 


\section{Competing interests}

The authors declare that they have no competing interests.

\section{Funding}

Supported by the National Natural Science Foundation of China (No. 81973871, 81804114), Clinical Research Plan of SHDC (No. SHDC2020CR1051B)

\section{Authors' contributions}

HW and WY conceived and designed the study. XZ executed the statistical methods and obtained ethical approval. HW, ZY, XW, ZS, and ZD drafted the article or revised it critically for important intellectual content. $\mathrm{HW}$ and $\mathrm{HZ}$ reviewed and edited the manuscript. Final approval of the version to be published was agreed upon by all authors.

\section{Acknowledgments}

We thank Pro. J He of Second Military Medical University for the help in statistics and Dr. M Zhang and Dr. W Tan of Shuguang Hospital Affiliated to Shanghai University of TCM for the radiological technical support. HW thanks for the inspiration and encouragement by the Chinese Luge team.

\section{Trial status}

The trial was registered at ClinicalTrials.gov on 13 November 2020 (identifier ChiCTR2000036515), and protocol version 2.1/20210120 is currently active. We started recruitment in February 2021 and it will be completed in November 2022.

\section{References}

1. Fardon DF, Milette PC. Nomenclature and classification of lumbar disc pathology: Recommendations of the Combined Task Forces of the North American Spine Society, American Society of Spine Radiology, and American Society of Neuroradiology. Spine. 2001;26:E93-113.

2. Sims JA, Moorman SJ. The role of the iliolumbar ligament in low back pain. Med Hypotheses. 1996;46:511-5.

3. Borenstein DG, O'Mara JW Jr, Boden SD, et al. The value of magnetic resonance imaging of the lumbar spine to predict low-back pain in asymptomatic subjects. J Bone Joint Surg Am. 2001;83A:1306-11.

4. Assendelft WJ, Morton SC, Yu El, Suttorp MJ, Shekelle PG. Spinal manipulative therapy for low-back pain. Cochrane Database Syst Rev. 2013;1:CD000447.

5. Kreiner DS, Hwang SW, Easa JE, et al. North American Spine Society. An evidence-based clinical guideline for the diagnosis and treatment of lumbar disc herniation with radiculopathy. Spine J. 2014 
Jan;14(1):180-91.

6. Reed WR, Cao DY, Long CR, Kawchuk GN, Pickar JG. Relationship between Biomechanical Characteristics of Spinal Manipulation and Neural Responses in an Animal Model: Effect of Linear Control of Thrust Displacement versus Force, Thrust Amplitude, Thrust Duration, and Thrust Rate. Evid Based Complement Alternat Med. 2013; 2013: 492039.

7. Cao DY, Reed WR, Long CR, Kawchuk GN, Pickar JG. Effects of thrust amplitude and duration of highvelocity, low-amplitude spinal manipulation on lumbar muscle spindle responses to vertebral position and movement. J Manipulative Physiol Ther. 2013 Feb;36(2):68-77.

8. He J, Xiao S, Wu Z, Yuan Z. Microendoscopic discectomy versus open discectomy for lumbar disc herniation: a meta-analysis. Eur Spine J. 2016 May;25(5):1373-81.

9. Pan M, Li Q, Li S, Mao H, Meng B, Zhou F, Yang H. Percutaneous Endoscopic Lumbar Discectomy: Indications and Complications. Pain Physician. 2020 Jan;23(1):49-56.

10. Wang H, Zhou Y, Li C, Liu J, Xiang L. Risk factors for failure of single-level percutaneous endoscopic lumbar discectomy. J Neurosurg Spine. 2015 Sep;23(3):320-5.

11. Shin EH, Cho KJ, Kim YT, Park MH. Risk factors for recurrent lumbar disc herniation after discectomy. Int Orthop. 2019 Apr;43(4):963-7.

12. Li Z, Yang H, Liu M, Lu M, Chu J, Hou S, Hou T. Clinical Characteristics and Risk Factors of Recurrent Lumbar Disk Herniation: A Retrospective Analysis of Three Hundred Twenty-One Cases. Spine (Phila Pa 1976). 2018 Nov 1;43(21):1463-1469.

13. Gerling MC, Leven D, Passias PG, Lafage V, Bianco K, Lee A, Lurie JD, Tosteson TD, Zhao W, Spratt KF, Radcliff K, Errico TJ. Risk Factors for Reoperation in Patients Treated Surgically for Lumbar Stenosis: A Subanalysis of the 8-year Data From the SPORT Trial. Spine (Phila Pa 1976). 2016 May;41(10):901-9.

14. Xiong YZ, Deng Z, Shen ZB, et al. Clinical observation on the treatment of inclusive lumbar intervertebral disc herniation with sci's great manipulation. Chinese J Trad Med Traum Orthop. 2018;26(9):41-5.

15. Akarawit Asawasaksakul K, Deshpande G, Choi, et al. Percutaneous Endoscopic Lumbar Diskectomy: Transforaminal Approach. In: Endoscopic Spine Surgery. 2nd edition [M]. New York: Thieme Medical Publishers, Inc.; 2018. pp. 11-6.

16. Pan M, Li Q, Li S, Mao H, Meng B, Zhou F, Yang H. Percutaneous Endoscopic Lumbar Discectomy: Indications and Complications. Pain Physician. 2020;23(1):49-56.

17. Shin BJ. Risk factors for recurrent lumbar disc herniations. Asian Spine J. 2014 Apr;8(2):211-5.

18. Yao M, Wang Q, Li Z, Yang L, Huang PX, Sun YL, Wang J, Wang YJ, Cui XJ. A Systematic Review of Cross-cultural Adaptation of the Oswestry Disability Index. Spine. 2016;41(24):1470-8.

19. Scudds RA. Pain outcome measures. J Hand Ther. 2001;14(2):86-90.

20. Camino Willhuber GO, Piuzzi NS. Straight Leg Raise T. 2020 Jul 31. In: StatPearls [Internet]. Treasure Island (FL): StatPearls Publishing; 2020 Jan-. PMID: 30969539. 
21. Harrison DE, Harrison DD, Cailliet R, Janik TJ, Holland B. Radiographic analysis of lumbar lordosis: centroid, Cobb, TRALL, and Harrison posterior tangent methods. Spine (Phila Pa 1976). 2001 Jun 1;26(11):E235-42.

22. Bambakidis NC, Feiz-Erfan I, Klopfenstein JD, Sonntag VK. Indications for surgical fusion of the cervical and lumbar motion segment. Spine. 2005;30(16 Suppl):2-6.

23. Tan MLS, Wee HL, Lee J, et al. The Short Form 36 English and Chinese versions were equivalent in a multiethnic Asian population[J]. J Clin Epidemiol. 2013;66(7):759-67.

24. Chinese Orthopaedic Association of Spinal Surgery Group; Chinese Orthopaedic Association of Orthopaedic Rehabilitation Group. Clinical practice guideline for diagnosis and treatment of lumbar disc herniation[J]. Zhong Hua Gu Ke Za Zhi. 2020;40(8):477-87.

\section{Tables}


Table 1

Flowchart of enrollment, intervention, data collection, and follow-up

\begin{tabular}{|c|c|c|c|c|c|c|c|}
\hline Timepoint & $\begin{array}{l}\text { Screening } \\
\text { Nisit } 0 \\
(0 \sim 3 \\
\text { Day } \\
\text { before } \\
\text { surgery) }\end{array}$ & $\begin{array}{l}\text { Visit } 1 \\
\text { (The } \\
\text { 2nd } \\
\text { Day } \\
\text { after } \\
\text { surgery) }\end{array}$ & $\begin{array}{l}\text { Visit } 2 \\
\text { (One } \\
\text { week } \pm \\
\text { two days } \\
\text { after } \\
\text { surgery) }\end{array}$ & $\begin{array}{l}\text { Visit } 3 \\
\text { (Four } \\
\text { weeks } \pm \\
5 d \text { after } \\
\text { surgery) }\end{array}$ & $\begin{array}{l}\text { Visit } 4 \\
\text { (Thirteen } \\
\text { weeks } \pm \\
1 \text { week } \\
\text { after } \\
\text { surgery) }\end{array}$ & $\begin{array}{l}\text { Visit } 5 \\
\text { (Forty- } \\
\text { eight } \\
\text { weeks } \pm 2 \\
\text { weeks } \\
\text { after } \\
\text { surgery) }\end{array}$ & $\begin{array}{l}\text { Visit } 6 \\
\text { (one } \\
\text { year } \pm 2 \\
\text { months } \\
\text { after } \\
\text { surgery) }\end{array}$ \\
\hline \multicolumn{8}{|l|}{ Enrollment } \\
\hline $\begin{array}{l}\text { [Eligibility } \\
\text { screen] }\end{array}$ & $x$ & & & & & & \\
\hline $\begin{array}{l}\text { [Informed } \\
\text { consent] }\end{array}$ & $x$ & & & & & & \\
\hline $\begin{array}{l}\text { [Random } \\
\text { allocation] }\end{array}$ & $x$ & & & & & & \\
\hline \multicolumn{8}{|l|}{ Assessments } \\
\hline [VAS scores] & $x$ & $x$ & $x$ & $x$ & $x$ & $x$ & $x$ \\
\hline [ODI] & $x$ & $x$ & $x$ & $x$ & $x$ & $x$ & $x$ \\
\hline $\begin{array}{l}\text { [Straight leg } \\
\text { raising } \\
\text { angle] }\end{array}$ & $x$ & $x$ & $x$ & $x$ & $x$ & $x$ & $x$ \\
\hline [SF-36v2] & $x$ & & & & & $x$ & $x$ \\
\hline $\begin{array}{l}\text { [Positive and } \\
\text { lateral X ray] }\end{array}$ & $x$ & $x$ & & $x$ & $x$ & $x$ & $x$ \\
\hline $\begin{array}{l}\text { [Dynamic X } \\
\text { ray] }\end{array}$ & $x$ & & & & $x$ & $x$ & $x$ \\
\hline [CT and MRI] & $x$ & $x$ & & & & $x$ & $x$ \\
\hline $\begin{array}{l}\text { Adverse } \\
\text { event }\end{array}$ & & $x$ & $x$ & $x$ & $x$ & $x$ & $x$ \\
\hline
\end{tabular}


Table 2

Inclusion and exclusion criteria

\section{Inclusion criteria}

20 to 70 years old

meet the diagnostic criteria of lumbar disc herniation with radiculopathy by Tian Wei et al.[24]

$\mathrm{ODI}>=10$

the course of a condition for more than 3 months and not improved with conservative therapy for more than 6 weeks

received no other treatment at the same time

voluntarily participant in this trial and signed the informed consent

Exclusion criteria

The presence of red flags or contraindications would put the participants at risk of injury.

tuberculosis, cancer, severe osteoporosis, acute infectious diseases, acute suppurative inflammation

severe cardiovascular, cerebrovascular, liver, kidney, hematopoietic, digestive system diseases or mental disorders

definite lumbar segmental instability or developmental lumbar spinal stenosis

current pregnancy or is prepared to get pregnant

People will also be excluded if they have the following:

misdiagnosis or mistaken inclusion

taking part in other clinical trials at the same time

violation of the protocol

compensable cases

self-decided exit

researchers consider he/she was not appropriate to participate in this study

\section{Figures}




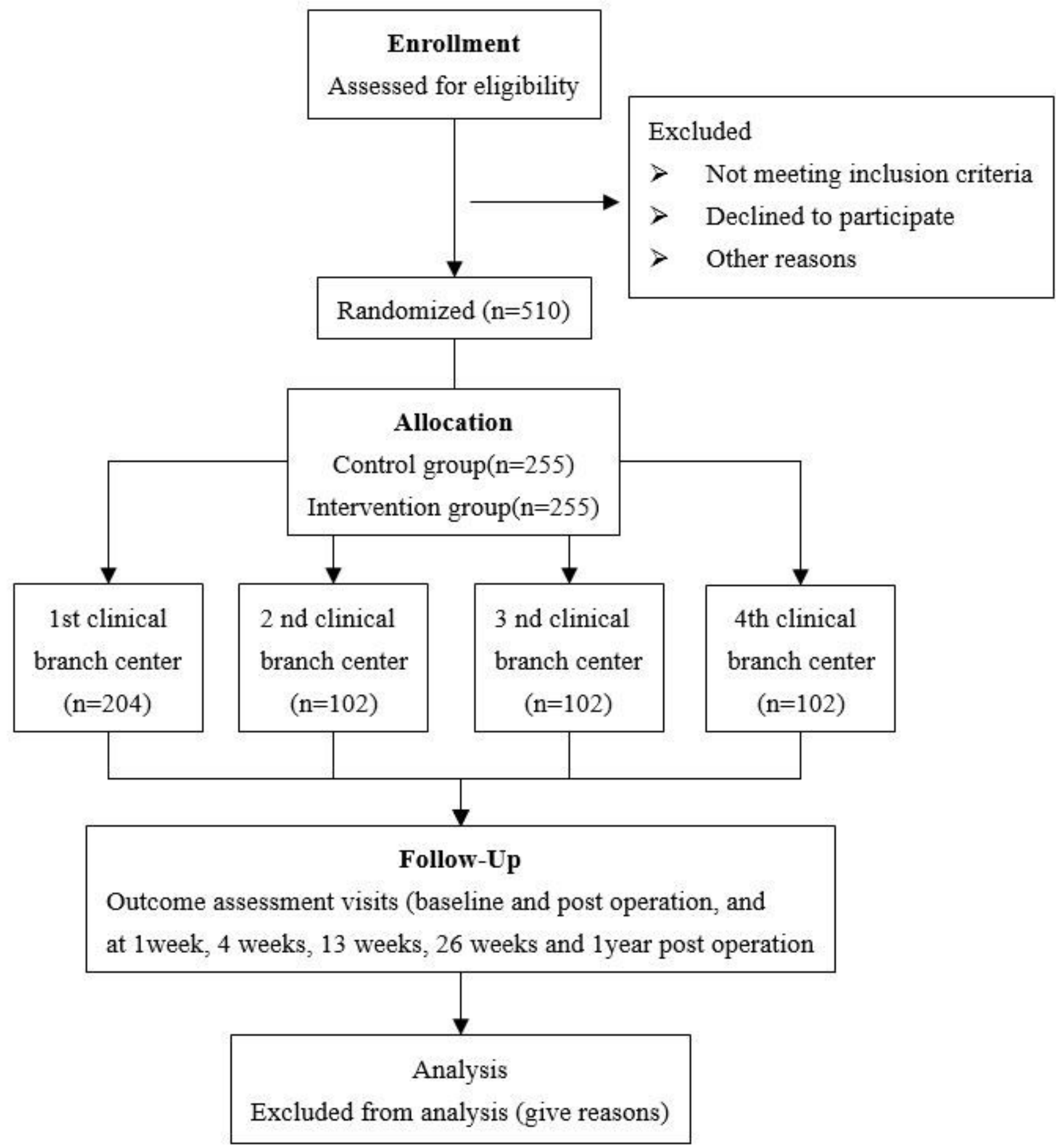

Figure 1

Study flow diagram of trial participation

\section{Supplementary Files}

This is a list of supplementary files associated with this preprint. Click to download. 
- 20210418SPIRITchecklist.doc

Page 19/19 